\section{Case Reports in Oncology}

\title{
A Case of Poorly Differentiated Large-Cell Neuroendocrine Carcinoma of the Cecum: A Rare Malignancy, with Review of the Literature
}

\author{
Andrew T. Mertz ${ }^{a}$ Michelle A. Ojemuyiwa ${ }^{b}$ \\ ${ }^{a}$ Uniformed Services University of the Health Sciences, Bethesda, MD, USA; ${ }^{b}$ Murtha \\ Cancer Center, Walter Reed National Military Medical Center, Bethesda, MD, USA
}

\section{Keywords}

Neuroendocrine carcinomas · Immunotherapy · Programmed death-ligand 1 .

Gastroenteropancreatic neuroendocrine tumors · Small-cell lung cancer

\begin{abstract}
Poorly differentiated neuroendocrine carcinomas (NECs) are rare tumors that can arise anywhere along the gastrointestinal tract. They often present in advanced stage and portend a poor prognosis when compared to adenocarcinomas of the same stage. Characterization of these tumors is best accomplished with tissue biopsy, as peripheral tumor markers commonly used in NECs are of little utility. Therapeutic strategies often involve chemotherapeutic regimens that have been used to treat small-cell lung cancer. Recent studies have shown that programmed death-ligand 1 (PD-L1) expression within poorly differentiated NECs is a poor prognostic indicator. However, PD-L1 expression may represent a possible target for immunotherapy drugs, often called checkpoint inhibitors, such as anti-PD-1 inhibitors.
\end{abstract}


 Oncology}

\section{Introduction}

Poorly differentiated neuroendocrine carcinomas (NECs) arising from the gastrointestinal (GI) tract are rare and have a very high malignant potential with a poor prognosis. These malignancies are diagnosed at roughly a rate of 1,000 cases annually, and therefore little is known regarding their tumor biology or cytogenetics [1]. Poorly differentiated NECs most commonly arise in the esophagus and large bowel; however, they may occur within virtually any portion of the GI tract to include the biliary system [2]. More importantly, more than $50 \%$ of the patients present with metastatic disease at the time of diagnosis $[2,3]$. This portends a poor prognosis for these patients, as the median overall survival (MOS) is only 5 months without intervention but can be augmented to a MOS of 8-20 months if some treatment modality is administered $[4,5]$.

\section{Case Report}

A 60-year-old female with a past medical history of adult-onset diabetes mellitus, scarlet fever, and uterine fibroids presented to her primary care manager with a 6-month history of progressive epigastric and right-lower-quadrant pain. A computed tomography (CT) scan of her abdomen was completed, revealing the presence of a mass in her right ascending colon. Her physician recommended surgical consultation; however, her consultation was initially delayed. After 1 week, she reported to the emergency department with acutely worsening abdominal pain accompanied by intractable nausea and vomiting. A CT scan of the abdomen was notable for bowel wall perforation and partial small bowel obstruction along with an $8 \times$ $12 \times 2 \mathrm{~cm}$ cecal mass with eccentric bowel wall thickening and associated pericecal lymphadenopathy (Fig. 1). She underwent emergent open right hemicolectomy followed by enterocolic anastomosis for the perforated cecal mass. Her pathology was notable for involvement of the entire thickness of the colon wall with extension into the subserosal fat. A total of 26 local regional lymph nodes were resected of which 17 were found to be involved. Immunohistochemical (IHC) stain was positive for synaptophysin, chromogranin, and SATB2 (Fig. 2) with a Ki-67 labeling index of 70\%. Based on the IHC stain assessment and the cellular morphology, she was diagnosed with high-grade large-cell NEC of the cecum. Postoperative CT of her chest, abdomen, and pelvis as well as magnetic resonance imaging of the brain were unremarkable for extensive disease. She also had a subsequent positron emission tomography scan that was unremarkable for systemic disease. She received 6 cycles of adjuvant chemotherapy with cisplatin and etoposide. Post-treatment imaging with full-body CT and positron emission tomography along with colonoscopic evaluation was negative for disease recurrence.

\section{Discussion}

Poorly differentiated NECs are rare cancers that can occur throughout the body. Histologically, poorly differentiated NECs are similar to small-cell lung cancer (SCLC) and thus are currently treated in a similar manner [6]. Studies estimate that these cancers are diagnosed at a rate of approximately 1,000 cases annually, with only $11 \%$ of the cases being located within the GI tract [1]. Approximately 3 out of 5 patients with poorly differentiated NECs present with metastatic disease at the time of their diagnosis and have poor prognostic out- 


\section{Case Reports in Oncology}

comes $[2,4]$. NECs are classified according to their mitotic count and Ki-67 proliferative index. Poorly differentiated NECs, otherwise called high-grade or G3 NECs, have a mitotic count of greater than $20 \times 10$ high-power fields and a Ki-67 level of greater than 20\% [7]. These tumors can be further stratified according to their specific histology that can be described as small cell, large cell, or mixed [7]. Studies have demonstrated that the Ki-67 level for this G3 subgroup is of clinical relevance, because tumors with a Ki-67 level of less than $60 \%$ are less responsive to established chemotherapy regimens for poorly differentiated NECs $[8,9]$. The vast range of Ki-67 indices within the G3 subgroup and the variation in the responses to standard chemotherapy regimens has raised questions as to whether there may be heterogeneity within the subgroup that could lead to further stratification and possibly even different treatments [6].

The presenting symptoms for these tumors depend primarily on the location. With the most common locations being the esophagus and large bowel, presenting symptoms include weight loss, pain, obstruction, bleeding, and in some cases even perforation $[2,10]$. The diagnosis of poorly differentiated NECs can be elusive with usual NEC tumor markers, such as chromogranin A and 5-hydroxyindoleacetic acid, being of little utility [4]. Tissue diagnosis is of critical importance when identifying these tumors, not only because they lack systemic markers, but also because the grade of differentiation is of prognostic significance [5].

Poorly differentiated NECs are traditionally managed using the same chemotherapy regimens as in SCLC, based upon their histologic similarity. Cisplatin or carboplatin and etoposide for 4-6 cycles is the standard treatment for limited-stage SCLC and is also used for limited-disease poorly differentiated NECs [11]. Extensive or metastatic disease is treated in the same manner; however, the duration is ill-defined as it is unclear as to whether more than 4 cycles of chemotherapy provide any additional survival benefit [11]. In the adjuvant setting, such as in the case of our patient, 4-6 cycles of platinum-etoposide chemotherapy is recommended with sequential radiation to follow in cases, such as rectum or cervical primary, where recurrence is thought to be higher [11]. For our patient, radiation was deferred, as the risk of increased toxicity was thought to be greater than the potential benefit. The patient was given 6 cycles of cisplatin-etoposide to provide prolonged systemic therapy without the increased risk of toxicity with locoregional radiation therapy.

Although systemic platinum-based therapy remains the most widely used treatment paradigm for these rare tumors, there have been very limited data on the appropriate second-line therapy. The use of immunotherapeutic agents that have shown promise in the treatment of other malignancies such as melanoma, non-small cell lung cancer, and renal cell carcinoma have peaked interest regarding their potential efficacy in poorly differentiated NECs. These agents exploit the interaction between PD-L1, expressed on the surface of cancer cells, and its receptor PD-1, located on the surface of T cells. The PD-1/PD-L1 interaction directly inhibits apoptosis of the tumor cell, by inhibiting $\mathrm{T}$ cells and blocking the antitumor immune response. Expression of PD-L1 is induced by proinflammatory molecules such as IFN- $\gamma$ and TNF- $\alpha$, which are produced by activated T cells. This localized immune suppression has been termed "adaptive immune resistance" because the tumor cells are effectively able to protect themselves by inducing PD-L1 in response to IFN- $\gamma$ produced by activated T cells [12]. PD-L1 expression has been evaluated in patients with small-cell neuroendocrine tumors and gastroenteropancreatic neuroendocrine tumors (GEP-NET). A single-institution study evaluated aberrant expression of PD-1 and PD-L1 by immunochemistry and RNAsequencing in small-cell NECs. In the 94 cases analyzed (61 pulmonary and 33 extrapulmonary), there was no considerable PD-L1 expression on tumor cells. However, PD-L1 expression was observed on tumor-associated macrophages and tumor-infiltrating lymphocytes 


\section{Case Reports in Oncology}

located in the stroma [13]. This is of significance because the tumor "microenvironment" may also be a target to consider for future clinical trials and anti-PD-L1 therapy. Suppression of the immune system by tumors in their "microenvironment" is why one such PD-L1 checkpoint inhibitor, nivolumab, has been shown to provide a durable SCLC antitumor activity response in both phase I and phase II trials [14]. To examine the role of PD-L1 expression in GEP-NET, a single-institution retrospective analysis was conducted evaluating the expression of PD-L1 in 32 patients with metastatic GEP-NET using immunohistochemistry. In this study, the expression of PD-L1 was associated with high-grade or G3 NEC subtypes as well as a significantly decreased MOS [15]. Mechanistically, this aberrant expression of PD-L1 on the NEC tumor cells provides the "adaptive immune resistance" necessary for immune system evasion, which makes the prospect of anti-PD-L1 agents as a new therapeutic modality especially exciting [15]

\section{Conclusion}

Poorly differentiated NECs of the GI tract are rare aggressive tumors that comprise less than $1 \%$ of the GI tumors. The most common location for these tumors along the GI tract is the esophagus and large intestine, but they can arise virtually anywhere, including all portions of the biliopancreatic system. The prognosis is poor because they often present in advanced stages. Treatment strategies are extrapolated from therapies employed in SCLC, as they have demonstrated an improved response rate and a prolonged MOS. Recent studies have shown that high-grade NECs express PD-L1, which may help them evade antitumor immune response mechanisms. New immunotherapy agents such as anti PD-L1 inhibitors present opportunities to capitalize on usual tumor immune evasion mechanisms such as the PD-L1 expression of high-grade NECs.

\section{Acknowledgements}

We thank Timothy Harkum, MD, from the Department of Pathology at Walter Reed National Military Medical Center.

\section{Statement of Ethics}

The authors have no ethical conflicts to disclose.

\section{Disclosure Statement}

The authors declare no conflicts of interest and have no disclosures.

\section{References}

Yao JC, Hassan M, Phan A, et al: One hundred years after 'carcinoid': epidemiology of and prognostic factors for neuroendocrine tumors in 35,825 cases in the United States. J Clin Oncol 2008;26:30633072. 
2 Brenner B, Tang LH, Klimstra DS, Kelsen DP: Small-cell carcinomas of the gastrointestinal tract: a review. J Clin Oncol 2004;22:2730-2739.

-3 Bernick PE, Klimstra DS, Shia J, et al: Neuroendocrine carcinomas of the colon and rectum. Dis Colon Rectum 2004;47:163-169.

-4 Sorbye H, Strosberg J, Baudin E, Klimstra DS, Yao JC: Gastroenteropancreatic high-grade neuroendocrine carcinoma. Cancer 2014;120:2814-2823.

-5 Shafqat H, Ali S, Salhab M, Olszewski AJ: Survival of patients with neuroendocrine carcinoma of the colon and rectum: a population-based analysis. Dis Colon Rectum 2015;58:294-303.

-6 Eads JR: Poorly differentiated neuroendocrine tumors. Hematol Oncol Clin North Am 2016;30:151-162.

7 Bosman FT, Carneiro F, Hruban RH, et al: WHO Classification of Tumors of the Digestive System. Geneva, WHO Press, 2010.

8 Sorbye H, Welin S, Langer SW, et al: Predictive and prognostic factors for treatment and survival in 305 patients with advanced gastrointestinal neuroendocrine carcinoma (WHO G3): the NORDIC NEC study. Ann Oncol 2013;24:152-160.

-9 Welin S, Sorbye H, Sebjornsen S, et al: Chemotherapy in poorly differentiated endocrine carcinoma after progression on first-line chemotherapy. Cancer 2011;117:4617-4622.

10 Brenner B, Shah MA, Gonen M, et al: Small-cell carcinoma of the gastrointestinal tract: a retrospective study of 64 cases. Br J Cancer 2004;90:1720-1726.

11 Strosberg JR, Coppola D, Klimstra DS, et al: The NANETS consensus guidelines for the diagnosis and management of poorly differentiated (high-grade) extrapulmonary neuroendocrine carcinomas. Pancreas 2010;39:799-800.

$12 \mathrm{He} \mathrm{J,} \mathrm{Hu} \mathrm{Y,} \mathrm{Hu} \mathrm{M,} \mathrm{Li} \mathrm{B.} \mathrm{Development} \mathrm{of} \mathrm{PD-1/PD-L1} \mathrm{pathway} \mathrm{in} \mathrm{tumor} \mathrm{immune} \mathrm{microenvironment} \mathrm{and}$ treatment for non-small cell lung cancer. Sci Rep 2015;5:13110.

-13 Schultheis AM, Scheel AH, Ozretić L, et al: PD-L1 expression in small cell neuroendocrine carcinomas. Eur J Cancer 2015;51:421-426.

14 Pedoeem A, Azoulay-Alfaguter I, Strazza M, et al: Programmed death-1 pathway in cancer and autoimmunity. Clin Immunol 2014;153:145-152.

15 Kim ST, Ha SY, Lee S, et al: The impact of PD-L1 expression in patients with metastatic GEP-NETs. J Cancer 2016;7:484-489. 


\section{Case Reports in Oncology}

\begin{tabular}{l|l}
\hline Case Rep Oncol 2016;9:847-853 \\
\hline DOI: 10.1159/000452655 & $\begin{array}{l}\text { C 2016 The Author(s). Published by S. Karger AG, Basel } \\
\text { www.karger.com/cro }\end{array}$ \\
\hline
\end{tabular}

Mertz and Ojemuyiwa: A Case of Poorly Differentiated Large-Cell Neuroendocrine Carcinoma of the Cecum: A Rare Malignancy, with Review of the Literature
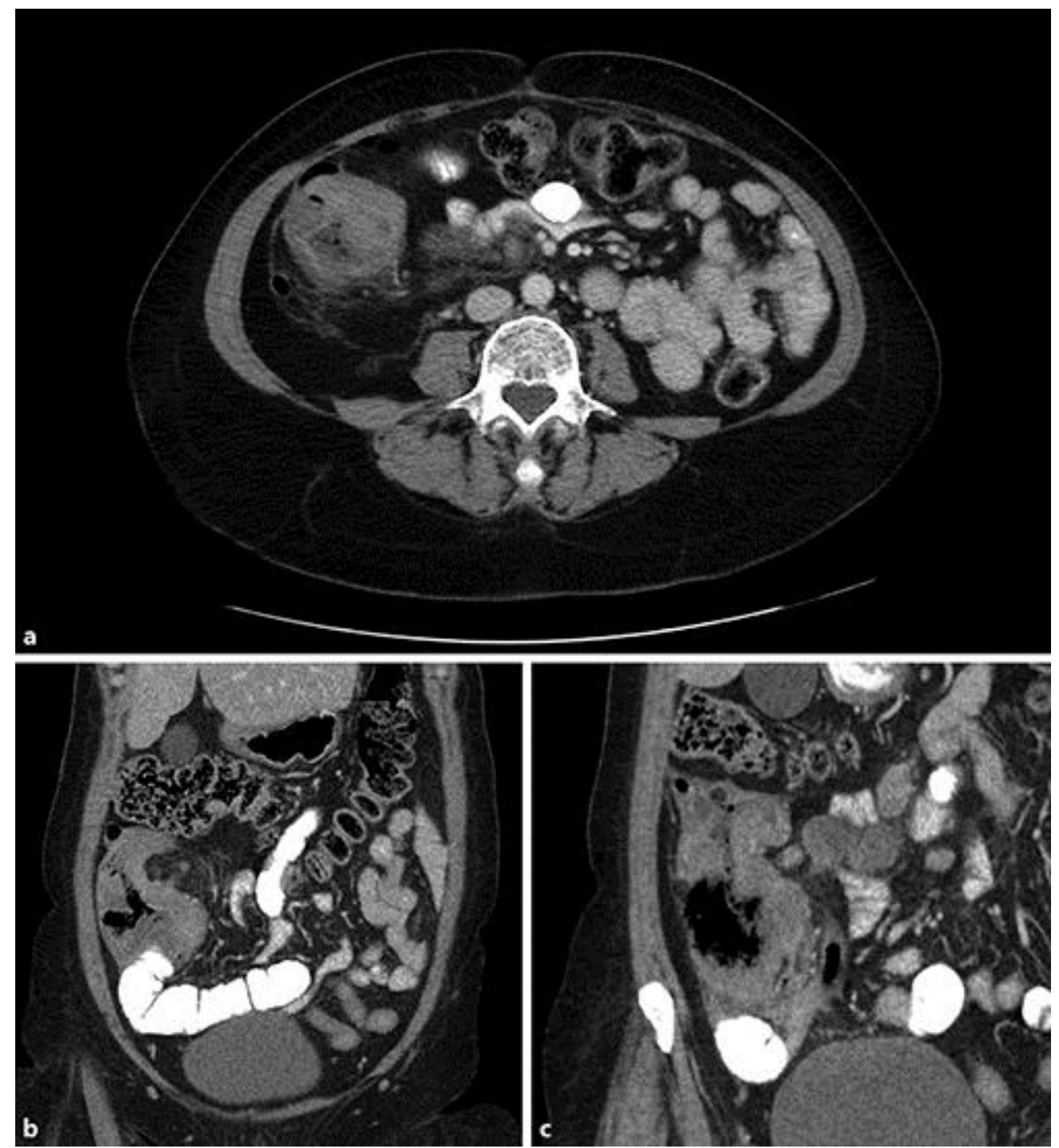

Fig. 1. a Contrast-enhanced CT axial view revealing a mass-like, eccentric bowel wall thickening of the cecum with associated bowel perforation. $\mathbf{b}$ Readily apparent transition point in the cecal area at the region of bowel wall thickening. c Heterogeneous cecal wall thickening with perforation as evidenced by an extraluminal gas pattern. 


\section{Case Reports in Oncology}
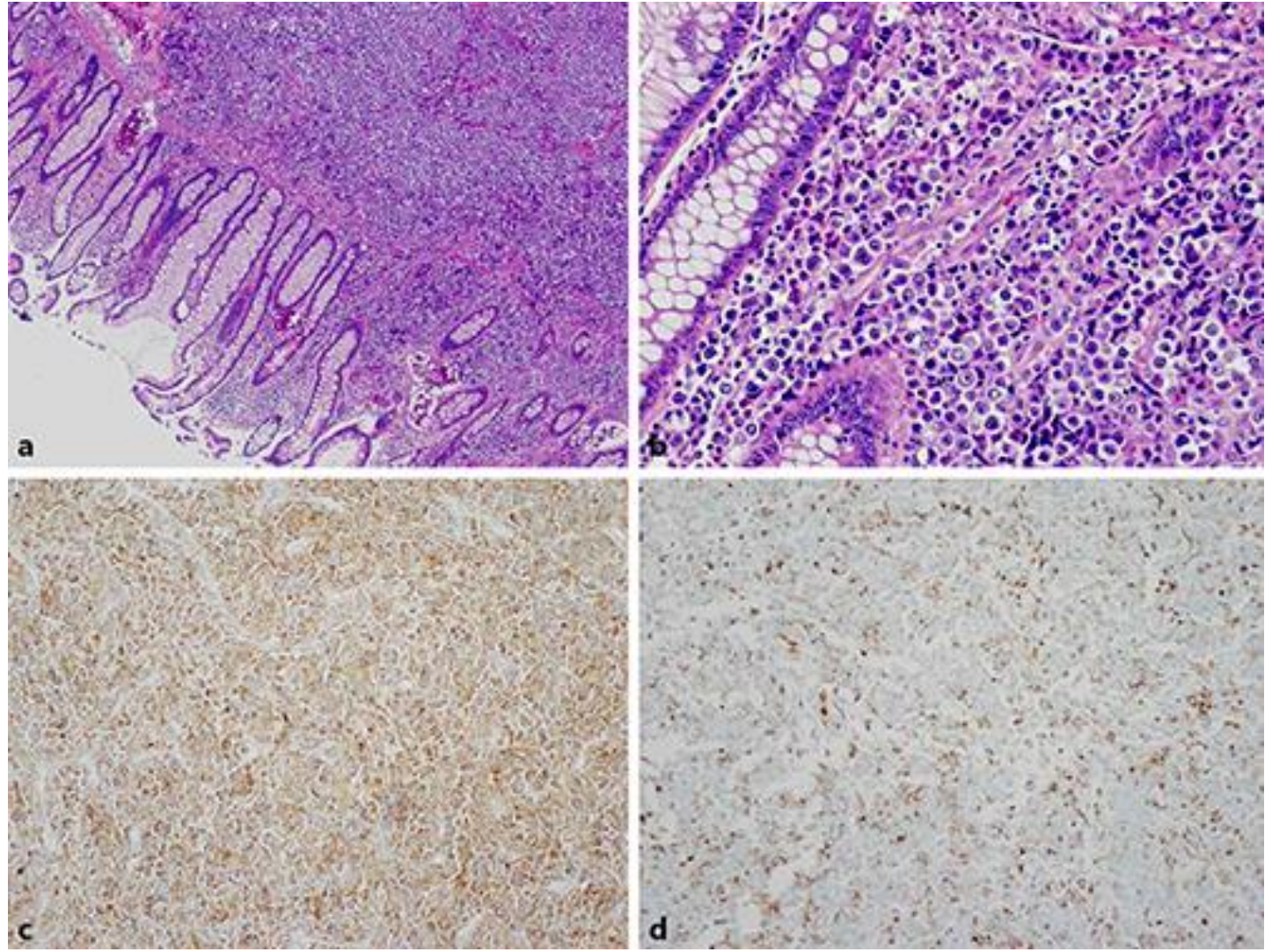

Fig. 2. a Low-power image showing diffuse infiltration of neoplastic cells into the colonic mucosa and submucosa. b High-power image showing nuclear pleomorphism, prominent nucleoli, scattered mitotic figures, and karyorrhectic debris with adjacent normal colonic epithelium. c Positive cytoplasmic staining with synaptophysin IHC stain. $\mathbf{d}$ Positive cytoplasmic staining with chromogranin IHC stain. 